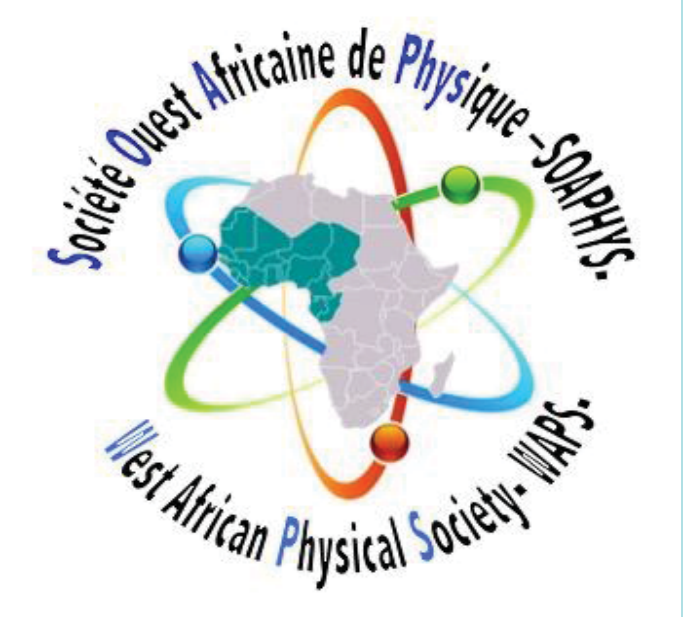

ISSN 2630-0958

Jourmal de Physique de la Soaphys J. P. Soaphys
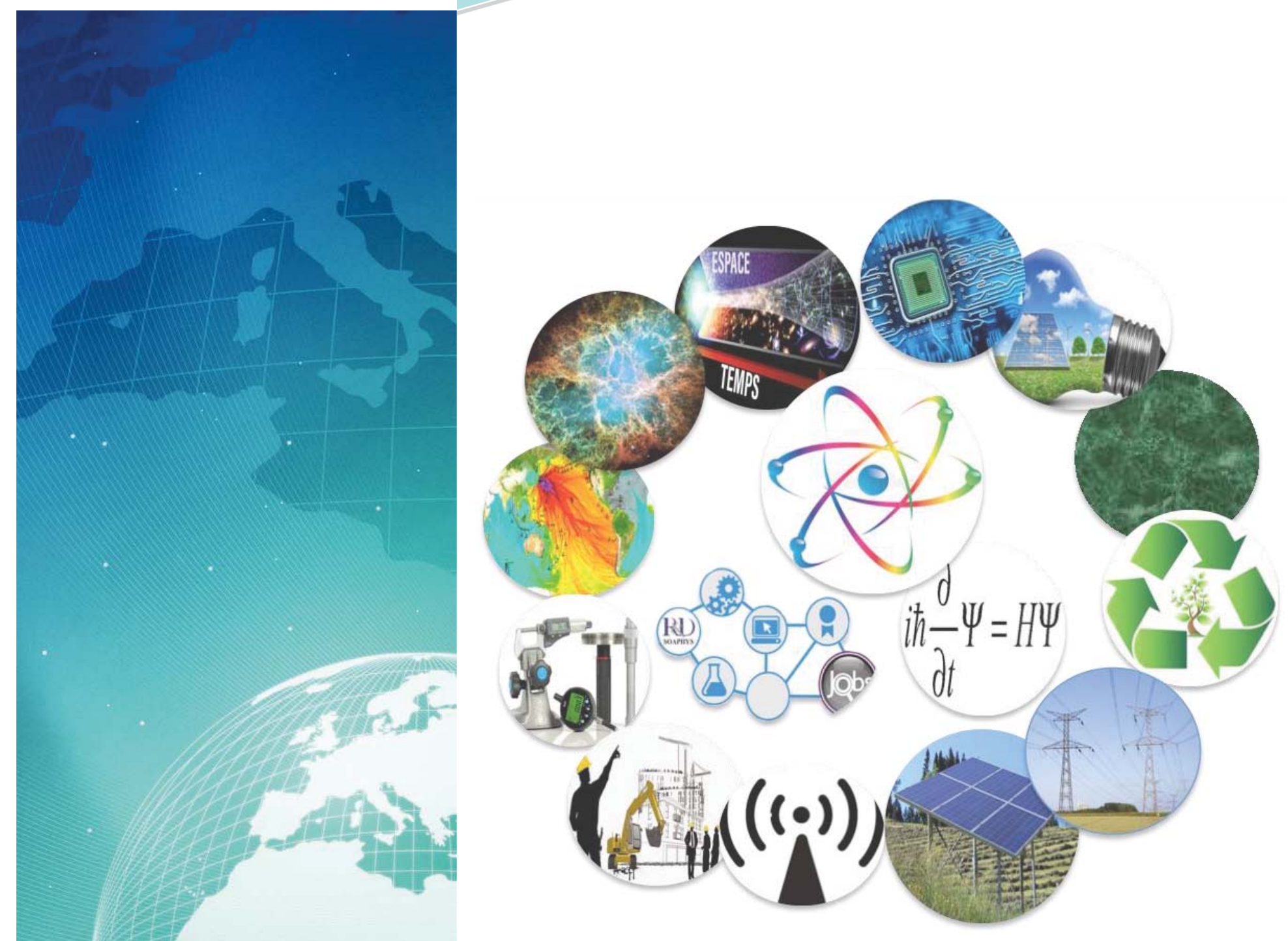

Volume 2, Numéro 1 - Décembre 2020 SOAPHYS ${ }^{\odot}$ 


\section{Journal de Physique de la Soaphys Volume 2, Numéro 1, Décembre 2020}

\section{Directeur de publication}

- Prof. Jean KOULIDIATI

\section{Comité scientifique}

- Prof. Jean CHABI OROU (Benin)

- Prof. Félix HONTINFINDE (Benin)

- Prof. Dieudonné Joseph BATHIEBO (Burkina Faso)

- Prof. Fréderic OUATTARA (Burkina Faso)

- Prof. Adama DIAWARA (Côte d'Ivoire)

- Prof. Vafi DOUMBIA (Côte d'Ivoire)

- Prof Abdramane BA (Mali),

- Prof. Badié DIOURTHE (Mali),

- Prof. Saïdou MADOUGOU (Niger)

- Dr. Haoua AMADOU, MC (Niger)

- Prof. Kossi NAPO (Togo)

- Prof. Magolmèèna BANNA (Togo)

- Prof. Oumar KA (Sénégal)

- Prof. Diouma KOBOR (Sénégal)

\section{Rédacteur en chef}

- Prof. Antoine BERE (Burkina Faso)

Rédacteur en chef adjoint

- Dr. Milohum Mikesokpo DZAGLI, MC, (Togo),

Secrétaire technique

- Dr. Sié Zacharie KAM, MA, (Burkina Faso) 


\section{Journal de Physique de la Soaphys Volume 2, Numéro 1, Décembre 2020}

\section{SOMMAIRE}

Analysis of extreme rains through climate indicators in the context of climate

$\mathrm{C} 20 \mathrm{~A} 1: 1-33$ change in southern Benin,

Hounvou et al.,

Comparative study of the power output of a mobile PV panel and a fixed PV

panel

Sène et al.,

Optimisation du rendement et de la température d'un capteur solaire plan à eau par simulation

Kanouté et al.,

Expérimentation et analyse thermique d'un concentrateur hémisphérique stationnaire sous les conditions climatiques à Ouagadougou, Burkina Faso Ouédraogo et al.,

Étude comparative de la digestion anaérobie entre pulpes de la pomme de cajou, bouse de vache et leur codigestion

Faye et al.,

Energy situation of Senegal: sub-sector of electricity

Ba et al.,

Low voltage grid connected three-phase inverter control with hybrid neuro-fuzzy Ndiaye et al.,

Assessment of different sands potentiality to formulate an effective thermal energy storage material (TESM).

Bagré et al.,

Évaluation de la dose aux patients lors de l'examen du thorax de face à Abidjan, Côte d'Ivoire

Konaté et al.,

Modélisation thermodynamique de combustion mono-zone de biodiesels dans un moteur diesel et estimation théorique des émissions potentielles

Dabilgou et al.,

Laboratory estimation of elemental and organic carbon emissions from advanced biomass stoves in Senegal

Kane et al.,

Exploitation du spectre de l'étoile HD149382 : approximation des paramètres physiques par une technique d'analyse spectrale Bado et al.,

Injection de puissance photovoltaïque au réseau électrique faible

Diaw et al.,

Assessing and modeling particulate pollution in the city of Ouagadougou

(Burkina Faso)

Ouarma et al., 


\title{
EXPLOITATION DU SPECTRE DE L'ÉTOILE HD149382 : APPROXIMATION DES PARAMÈTRES PHYSIQUES PAR UNE TECHNIQUE D'ANALYSE SPECTRALE
}

\author{
Bado Fabrice *, Kam Sié Zacharie, Ouédraogo Tongonmanegde Léonard, Sougoti Moussa, Zongo Sidiki, \\ Koulidiati Jean, and Béré Antoine
}

Laboratoire de Physique et de Chimie de l'Environnement, Université Joseph KI-ZERBO, Ouagadougou, Burkina Faso

\author{
*fabricebado726@gmail.com
}

\section{INFOS SUR L'A R T I C L E}

Historique de l'article :

Reçu le : 20 juin 2020

Reçu en format révisé le : 02 février 2021

Accepté le : 04 février 2021

Mots-Clés : HD149382 ; Méthode indirecte; Paramètres physiques

Keywords : HD149382 ; Indirect method ; Physical parameters

\begin{abstract}
R É S U M É
La détermination des paramètres physiques tels que la température effective, la gravité de surface, la luminosité, et le rayon d'une étoile nécessite une analyse adéquate de ses relevés spectroscopiques. Dans cet article, l'objectif est de déterminer ses paramètres physiques pour l'étoile HD149382 à partir de son spectre. Notre méthode indirecte consiste à déterminer la largeur à mi-hauteur des profils des raies He I $\lambda 471.362 \mathrm{~nm}$, He I $\lambda 501.605 \mathrm{~nm}$, et He I $\lambda 667.872 \mathrm{~nm}$ qui présentent un profil en cloche et qui résultent de la vie radiative au sein de l'étoile et de la durée de vie des particules suite aux collisions. Nous utilisons une courbe de Gauss (Gaussienne) pour la modélisation des raies de l'hélium. Cette modélisation à consister dans un premier temps à extraire les raies considérées et à les modéliser à partir du logiciel Matlab. Notre méthode est principalement basée sur la détermination de la température effective, le rayon, la gravité de surface et la luminosité de l'étoile HD149382 par effet Doppler grâce à la largeur à mi-hauteur du profil Gaussien de la raie d'absorption modélisée. Les résultats obtenus montrent que la température effective, la gravité de surface, la luminosité, et le rayon ont des valeurs comprises respectivement entre 33 427-40 051.7 K, 4.979-6.764 cm.s $\mathrm{s}^{-2}, 86.821-86.675 \mathrm{erg} / \mathrm{K}^{3} / \mathrm{s}$ et $3.34 \mathrm{R}_{\odot}-10 \mathrm{R}_{\odot}$ et que l'étoile HD149382 est une étoile naine de type sdB.
\end{abstract}

\begin{abstract}
A B S T R A C T
The determination of physical parameters such as effective temperature, surface gravity, luminosity, and radius of a star requires an adequate analysis of its spectroscopic readings. In this paper, the main aim is to determine the physical parameters of the star HD149382 from its spectrum. Our indirect method consists in determining the half-height width of the profiles of the He I $\lambda 471.362 \mathrm{~nm}, \mathrm{He}$ I $\lambda 501.605 \mathrm{~nm}$, et He I $\lambda 667.872 \mathrm{~nm}$ lines which have a bell-shaped profile and which result from the radiative life within the star and the lifetime of the particles after collisions. We use a Gaussian curve for helium line modeling. This modeling consists first in extracting the considered lines and modeling them from the Matlab software. Our method is mainly based on the determination of the effective temperature, the radius, the surface gravity and the luminosity of the star HD149382 by Doppler Effect thanks to the half-height width of the Gaussian profile of the modeled absorption line. The results obtained show that the effective temperature, the surface gravity, the luminosity, and the radius have values ranging respectively between $33427-40051.7 \mathrm{~K}, 4.979-6.764 \mathrm{~cm} . \mathrm{s}^{-2}$, 86.821-86.675 $\mathrm{erg} / \mathrm{K}^{3} / \mathrm{s}$ et $3.34 \mathrm{R}_{\odot}-10 \mathrm{R}_{\odot}$ and that the star HD149382 is a dwarf star of sdB type.
\end{abstract}

\section{INTRODUCTION}

L'étude des étoiles sous naines de types B, subdwarfs sdBs, contribue à la compréhension de la formation et de la morphologie des galaxies.
Les sdBs ont une magnitude moyenne dans la bande $\mathrm{V}$ $4<\mathrm{Mv}<6$ [1]. Avec leur nombre elles contribuent à la brillance des galaxies elliptiques en particulier dans l'ultra-violet. Elles passent la majeure partie de leur vie sur la branche extrême gauche de la branche horizontale (EHB). La spectroscopie est une des méthodes efficaces 
pour sonder ces types d'étoiles. Les raies spectrales observées, résultent de la transition discrète d'un état atomique à l'autre. Ces raies sont caractérisées par la longueur d'onde centrale, largeur et profondeur, de même que par les opacités de type liée-liée, liée-libre, libre-libre ou de la diffusion.

Notre cible HD149382 est une sdB difficile à observer à l'œil nu, elle est située dans la constellation d'Ophiuchus qui a une magnitude apparente de $+8,943$ [2].

Selon le data release II du consortium de collaboration Gaia [3], et se basant sur les mesures de parallaxe, l'étoile est à une distance d'environ 77 pc soit $\sim 251$ a.l.

Les paramètres physiques de HD149383 sont bien décrits par S. Geier et al. [4].

La Température effective ( $\left.\mathrm{T}_{\text {eff }}\right)$, la gravité de surface (log $\mathrm{g})$, la luminosité $(\log \mathrm{L})$, et le rayon $\left(\mathrm{R}_{\odot}\right)$ sont des paramètres physiques fondamentaux indispensables pour la caractérisation d'une étoile. En effet, ils permettent de déterminer l'évolution, la position de l'étoile sur le diagramme Hertzsprung-Russel (diagramme HR) et de tester les modèles théoriques [5,6]. Les valeurs exactes de ses paramètres physiques fondamentaux sont nécessaires pour déterminer l'évolution et la position des étoiles sur un diagramme HR (Figure 1). Développé par HertzsprungRussell et Henry Norris Russell ; le diagramme HR donne un schéma évolutif des étoiles dans un amas. Dans ce diagramme est indiquée la luminosité d'un ensemble d'étoiles en fonction de leur température effective ou leur classe spectrale.

Les étoiles extrêmes de la branche horizontale (EHB) sont des sous-naines de type $\mathrm{O}$ et $\mathrm{B}(\mathrm{sdB} / \mathrm{sdO})$ [5]. La branche horizontale (HB) est un nouvel état d'équilibre pour l'étoile après la séquence principale. Ces étoiles, ayant souvent évoluées au-delà de ce stade [7], sont considérées comme des étoiles brûlant un noyau d'hélium avec des enveloppes d'hydrogène très minces et ayant des masses d'environ $0.5 \mathrm{M}_{\odot} \quad\left(\mathrm{M}_{\odot}\right.$ est la masse solaire et vaut $1.9884 * 10^{30} \mathrm{~kg}$ ) [5] car elles ont évolué et continuent de transformer de l'hélium dans leur cœur. Les étoiles bleues de la Branche Horizontale (BHB) présentent souvent des anomalies d'abondances de certains éléments chimiques dans leur atmosphère [8]. Les sdO sont plus chaudes avec des températures de surface supérieures à $40000 \mathrm{~K}$. On les retrouve dans le diagramme Hertzsprung-Russel (Fig. 1).

L'effet Doppler ou élargissement Doppler est lié au mouvement thermique. La longueur d'onde des raies spectrales dépend de la vitesse de la source et de l'observateur. Cet effet crée un décalage des raies observées du fait qu'il affecte les ondes lumineuses. Cela se produit lorsqu'il existe un mouvement relatif entre une source d'ondes et l'observateur. Les raies dans ce cas, sont représentées par une Gaussienne et notre analyse méthode est basée sur la détermination de la largeur à mi-hauteur des raies modélisées. Les paramètres stellaires de l'étoile varient en fonction des réactions thermonucléaires au sein d'une étoile [4]. Pour la détermination de ces paramètres, il existe diverses méthodes indirectes telles que les relations de calibration, les grilles de calibration photométrique, la relation température effective-couleur, la méthode du flux infrarouge, le flux convenable, la proportion des lignes spectrales, le diagnostic des lignes métalliques, l'ajustement spectral global, et la connaissance du spectre observé à travers le profile Balmer, les raies d'hélium [10].

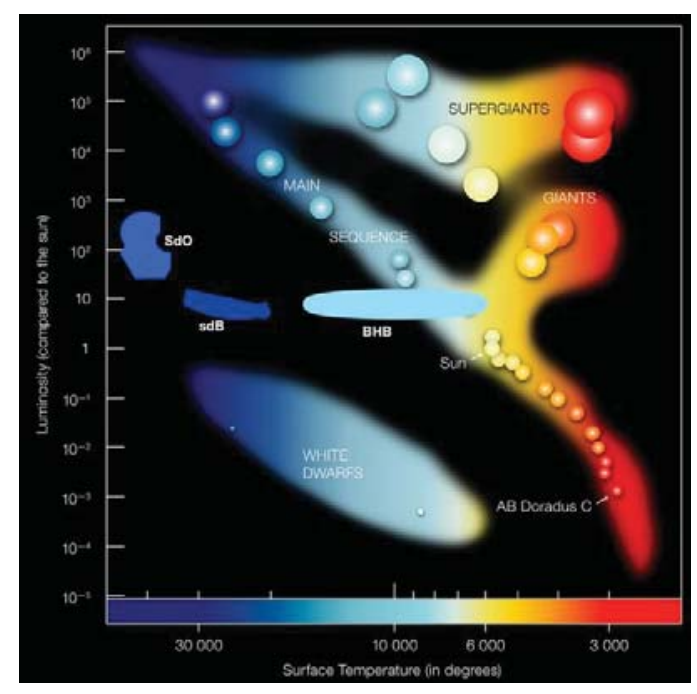

Figure 9 : Diagramme HR montrant la position des sdB et sdO et BHB, Figure adaptée de ESO/José Francisco (josefrancisco.org) une description similaire des types étoiles dans [9]

\section{MÉTHODES}

Dans ce travail, nous utilisons une méthode basée sur une analyse spectrale qui consiste à déterminer par modélisation la largeur à mi-hauteur de la raie du spectre d'absorption de l'hélium pour la caractérisation de l'étoile HD 149382.

Dans la méthode directe, les paramètres physiques cherchés sont obtenus par une mesure physique directe sur l'étoile : des observations. Dans le cadre de ce travail, nous n'utilisons pas de modèle d'atmosphère. Cependant nous utilisons une approche simple de modélisation des raies des atomes d'hélium pour déterminer nos paramètres physiques à partir de la détermination des largeurs à mihauteur des modèles de nos raies.

\subsection{Acquisition des données}

Les données analysées, issues des archives de l'observatoire Mauna Kea (www.cadc-ccda.hia-iha.nrccnrc.gc.ca), ont été utilisées. Les observations ont été réalisées en 2010 entre les mois de janvier à aout au cours du projet 10AF18 du CFHT. Les données obtenues au cours de ce projet sont finalement publiées sous forme de spectres bruts ou réduits afin de permettre d'autres études.

Ces données sont acquises par le spectrographe ESPaDOnS du télescope Canada France Hawaï. 
ESPaDOnS est un spectro-polarimètre ayant une résolution de l'ordre de 65000 et une bande spectrale allant de 370 à $1050 \mathrm{~nm}[11,12]$. Les observations présentées dans cette étude sont celles de l'acquisition du spectre brut de l'étoile HD149382 qui ont eu lieu le 27 janvier 2010 entre $14: 30: 57.690$ et $14: 44: 32.723$. Pour l'extraction, la modélisation des raies et la représentation du profile spectral du corps noir de l'étoile HD149382, nous avons utilisé les spectres normalisés et non normalisés obtenus grâce au logiciel Libre ESpRIT à travers le programme upena [12].

\subsection{Modélisation et analyse des données}

Nos modélisations ont été faites sous Matlab et Python. Les valeurs des longueurs d'ondes utilisées dans la modélisation sont extraites de la base de données des spectres atomiques du National Institute of Standards and Technology (NIST) (www.nist.gov/pml/atomic-spectradatabase).

Allant de l'idée d'un corps noir stellaire, la modélisation des différentes raies a été faite à partir de la fonction Gaussienne donnée dans l'équation 1 :

$G(x)=\frac{1}{\Delta \lambda_{D} \sqrt{(\Pi)}} \exp \left(\frac{-\left(\lambda-\lambda_{0}\right)}{\Delta \lambda_{D}}\right)^{2}$

\subsection{Détermination des paramètres physiques}

La température effective est un paramètre atmosphérique nécessaire pour la caractérisation d'une étoile ; elle est définie par l'équation 2 :

$$
T=\frac{m c^{2}}{8 \ln (2) k_{b} \lambda^{2}} F W H M^{2}
$$

avec $m$ la masse de l'espèce chimique considérée ; $c$ la vitesse de la lumière dans le vide; $k_{b}$ la constante de Boltzmann; $\lambda$ la longueur d'onde de l'espèce chimique considérée ; FWHM (Full Width and Half Maximum) la largeur à mi-hauteur.

La pression photosphérique de l'atmosphère stellaire d'une étoile encore appelé la gravité de surface est exprimée par la relation 3 :

$\log (g)=\log (G)+\log \left(M_{\odot}\right)-2 \log \left(R_{\odot}\right)$

avec $G$ la constante gravitationnelle, $\mathrm{M}_{\odot}$ et $\mathrm{R}_{\odot}$ sont respectivement la masse et le rayon de l'étoile exprimés en unités solaire.

La masse considérée est comprise entre $0.29 \mathrm{M}_{\odot}$ et 0.53 M๑ [4] pour l'étoile HD149382. La luminosité L est donnée par l'Équation 4 :

$$
\log (L)=4 \log \left(T_{\text {eff }}\right)+2 \log \left(R_{\odot}\right)+\log \left(4 \pi \sigma_{S B}\right)
$$

avec $\sigma_{S B}$ la constante de Stefan-Boltzmann.

\section{RÉSULTATS ET DISCUSSION}

Le spectre de l'étoile HD149382 est acquis sur une bande spectrale allant des Ultra-Violet (UV) au proche infrarouge. Dans le domaine du visible, on observe la présence des raies d'absorptions correspondant à celles de l'hydrogène à $410.2 \mathrm{~nm}, 434.1 \mathrm{~nm}$ et celles de l'hélium à $402.7 \mathrm{~nm}, 438.8 \mathrm{~nm}$ et $447.8 \mathrm{~nm}$. Dans cette région du spectre, ces raies sont plus intenses, traduisant ainsi la transformation de l'hydrogène en hélium dans l'atmosphère de l'étoile comme indiqué dans la Figure 2.

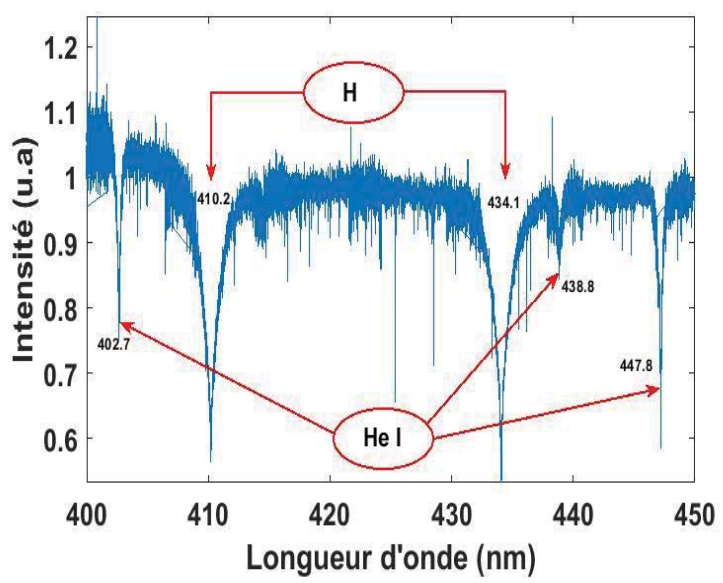

Figure 10 : Présence de l'hydrogène (H) et de l'hélium (HeI) dans le spectre de HD149382 compris entre $400 \mathrm{~nm}$ à $450 \mathrm{~nm}$ qui prouvent la présence de $\mathrm{H}$ et He à différentes longueurs d'ondes.

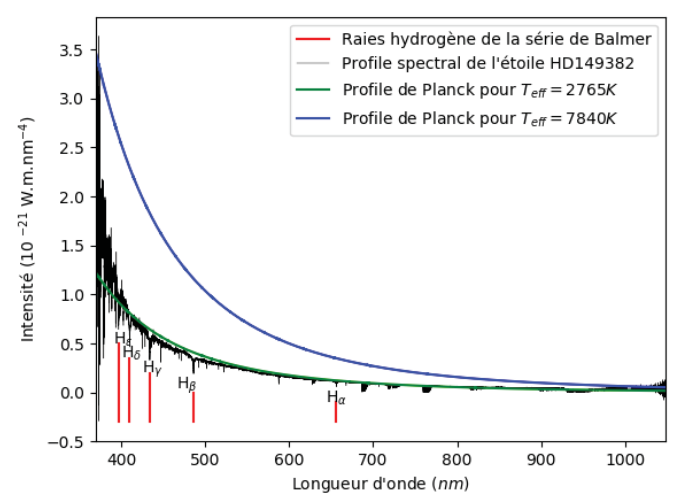

Figure 11 : Figure présentant le profil du corps noir de l'étoile HD149382 (en noire) ainsi que le profil de Planck pour $2765 \mathrm{~K}$ (en vert) et $7840 \mathrm{~K}$ (en bleu) en considérant le minimum et le maximum des longueurs d'ondes et la loi de Wien.

La largeur à mi-hauteur (FWHM) est déduite par un ajustement de type Gaussien des raies modélisées, présentées en figures 4,5 , et 6 . Les températures déduites sont présentées dans le tableau 1. Ces températures sont comparables à celles trouvées dans les travaux de S. Geier et al. [4], U. Heber [9] et E. Niemczura et al. 10] ; lesquelles raies présentaient les températures $35500 \mathrm{~K} \pm$ $500,40000 \mathrm{~K}$ et $35000 \mathrm{~K} \pm 2000$ respectivement. On obtient $12.82 \%, 7.67 \%, 5.84 \%$ et $0.13 \%, 4.47 \%, 16.43$ $\%$ et $9.18 \%, 4.49 \%$ comme incertitudes pour les différentes raies modélisées. 
Tableau 1 : Valeur de FWHM et de $T_{\text {eff }}$ des raies He I modélisées

\begin{tabular}{lcc}
\hline Raies & FWHM (nm) & Teff $_{\text {eK) }}$ \\
\hline He I 667.875 & 0.095 & 40051.7 \\
\hline He I 471.362 & 0.057 & 38213.3 \\
\hline He I 501.605 & 0.066 & 33427.2 \\
\hline
\end{tabular}

En plus des raies $H_{\alpha}, H_{\beta}, H_{\delta}, H_{\varepsilon}$, et $H_{\gamma}$, de la série de Balmer (figure 7), et des raies d'hélium HeI, on observe la présence des raies de l'hélium HeII [3, 9], du soufre SIII et de l'oxygène qui se dédoublent à $\lambda 425.378 \mathrm{~nm}$ (longueur d'onde). La présence des raies de Balmer dans le spectre de l'étoile donne une idée de sa température. En effet, lorsque la température est très élevée, la majeure partie des atomes d'hydrogène est ionisée et les raies d'absorption formées sont moins intenses.

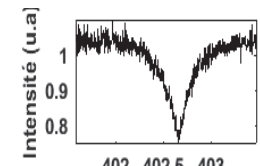

402402.5403
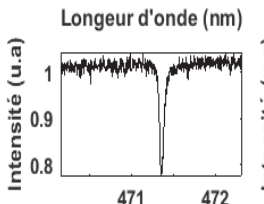

Longeur d'onde $(\mathrm{nm})$

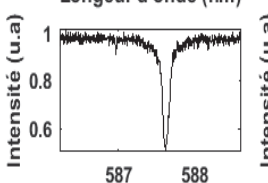

Longeur d'onde $(\mathrm{nm})$

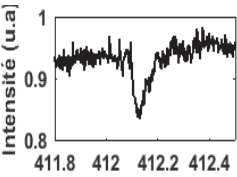

Longeur d'onde $(\mathrm{nm})$

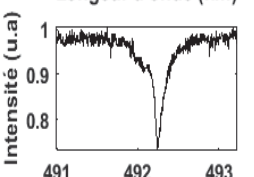

Longeur d'onde $(\mathrm{nm})$

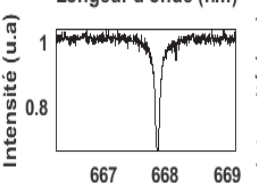

Longeur d'onde $(\mathrm{nm})$

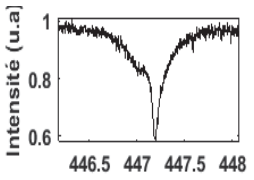

Longeur d'onde $(\mathrm{nm})$

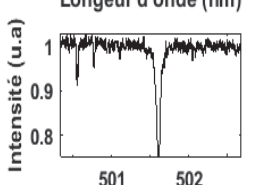

Longeur d'onde (nm)

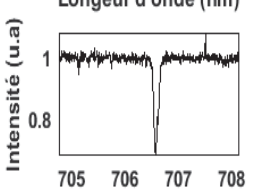

$705 \quad 706 \quad 707 \quad 708$
Longeur d'onde $(\mathrm{nm})$
Figure 12 : Raies de He I extraite du spectre de HD149382. La première rangée, de gauche à droite, montre respectivement les raies à $\lambda 402.6, \lambda 412.08, \lambda 447.2$; pour la deuxième rangée nous avons les raies à $\lambda 471.31, \lambda 492.2, \lambda 501.6$; la troisième rangée présente les raies à $\lambda 587.5, \lambda 667.8$ et $\lambda 706.59$.

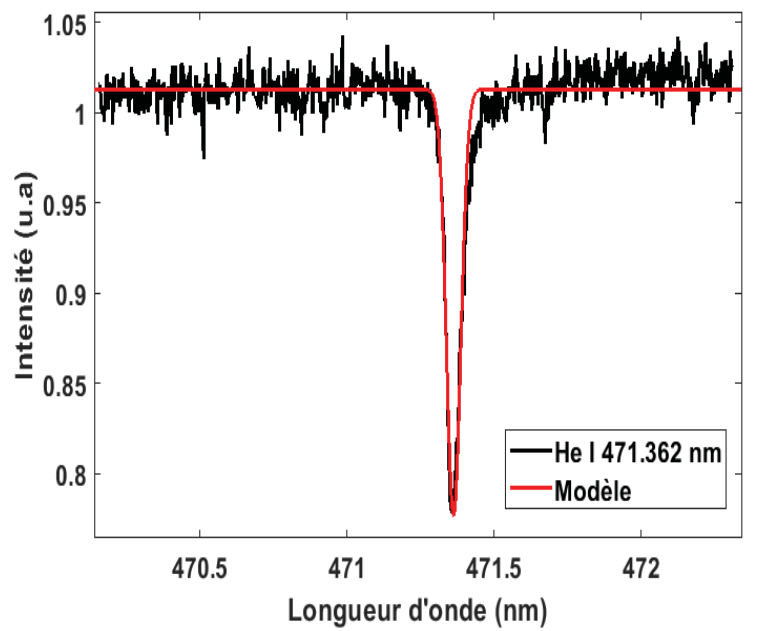

Figure 13 : Modélisation de la raie He I $\lambda 471.362$. La raie est représentée en noire et le modèle en rouge.

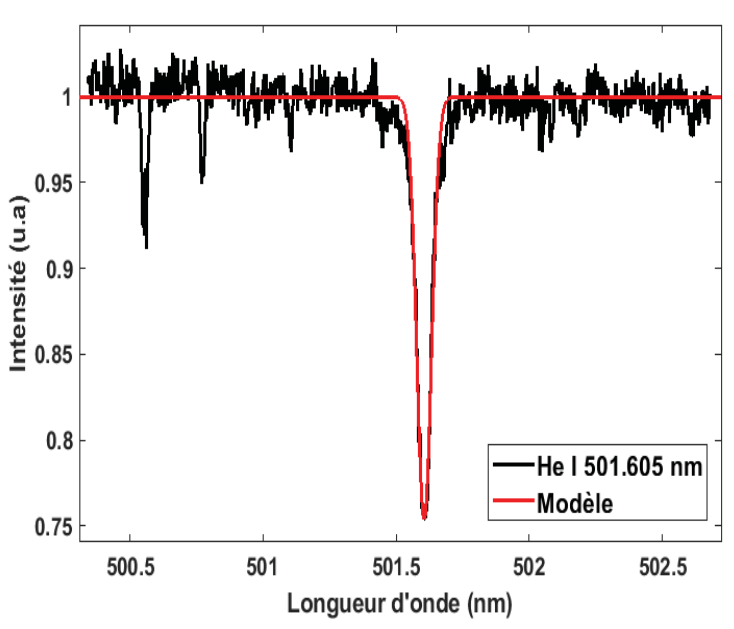

Figure 14 : Modélisation de la raie HeI $\lambda 501.605 \mathrm{~nm}$. La raie est représentée en noire et le modèle en rouge.

Les écarts entre nos raies et nos modèles peuvent être expliqués par le bruit présent dans le spectre de l'étoile, de même que le profil Gaussien ne prend pas en compte les ailes des raies considérées.

La loi de Wien permet, d'une part, de relier la température $\mathrm{T}$ (en $\mathrm{K}$ ) à la longueur d'onde $\lambda_{M}(\mathrm{~m})$ par la relation suivante : $\lambda_{M} T=2.9 * 10^{-3} \mathrm{mK}$. D'autre part, elle est caractéristique d'un corps noir. Selon cette loi, la longueur d'onde à laquelle un corps noir émet le plus de flux lumineux énergétique est inversement proportionnelle à sa température. Cette loi ne permet pas d'obtenir la température effective de l'étoile HD149382 (Figure 2).

Les raies sont sensibles à la valeur de la gravité de surface, plus la surface de gravité augmente plus la raie est élargie au niveau des ailes. De même toutes les raies modélisées présentent un cœur profond avec des ailes faibles, figures 4,5 et 6 .

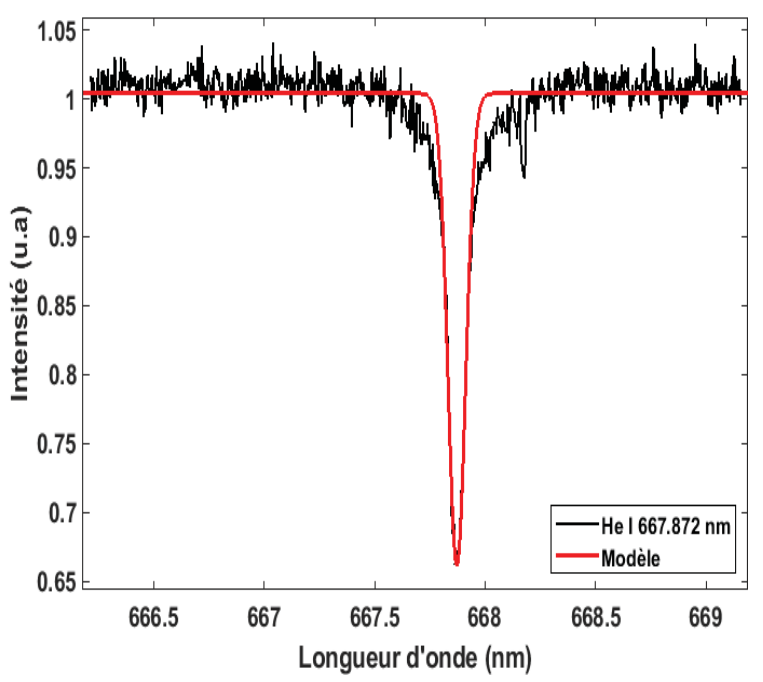

Figure 15 : Modélisation de la raie He I 2667.875 . La raie est représentée en noire et le modèle en rouge. 


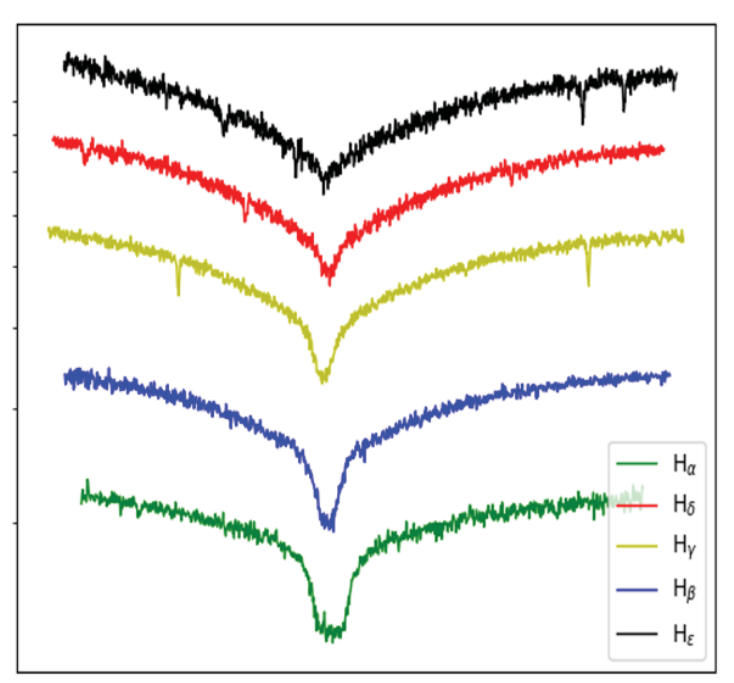

Figure 16 : Superposition des raies hydrogène de la Série de Balmer montrant la profondeur des différentes raies. On note que les raies $H_{\alpha}$ et $H_{\beta}$ sont les plus profondes.

Des relations 4 et 5 , les paramètres physiques tels que le rayon $\mathrm{R}_{\odot}$, la gravité de surface $\log g$, et une luminosité log $L$, dont les valeurs sont respectivement comprises entre

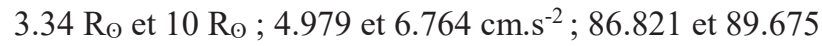
$\mathrm{erg} / \mathrm{K}^{3} / \mathrm{s}$. Utilisant des modèles d'atmosphères, T. Lisker et al. [15] trouvent une surface de gravité comprise entre 4.8 et $6.0 \mathrm{~cm} . \mathrm{s}^{-2}$; U. Heber et al. [16] trouvèrent un $\log g=5.5$ avec une température effective de $35000 \mathrm{~K}$ pour l'étoile HD149382. Par ailleurs, [4] ont comme paramètres $35500 \pm 500 \mathrm{~K}$ pour la $T_{\text {eff }}$ et $\log g=5.80 \pm 0.05$ pour la gravité. [14], quant à eux trouve $T_{\text {eff }}=(35000 \pm 2000) \mathrm{K}$ et un $g_{s}=10^{5.5 \pm 0.3} \mathrm{~cm} \cdot \mathrm{s}^{-2}$. En 1975, [13] à travers leur étude sur la nature des étoiles sous-naine de type $\mathrm{B}$ à partir de la sous-naine OB HD149382 trouvent pour ladite étoile une $T_{\text {eff }}=40000 \mathrm{~K}$ et un $\log g=5.8$ avec une déficience en Hélium d'un facteur 10 comparativement aux étoiles de même type de population I.

\section{CONCLUSION}

Nous avons effectué une analyse spectroscopique de 1'étoile HD149382. À travers la modélisation des raies de l'hélium intégrant des paramètres physiques de l'étoile. Les résultats obtenus confirment que l'étoile HD149382 est de type sdOB, avec une atmosphère riche en hélium dont la température effective est comprise entre 33427.2 et $40051.7 \mathrm{~K}$. Les valeurs de la gravité de surface varient de 4.979 à $6.764 \mathrm{~cm} . \mathrm{s}^{-2}$ tandis que la luminosité est de l'ordre de $86.821-89.675 \mathrm{erg} / \mathrm{K}^{3} / \mathrm{s}$. De plus, nous avons obtenu une approximation du rayon de l'étoile HD149382 compris entre $3.34 R_{\odot}$ et $10 R_{\odot}$. Nos profiles des raies ne reproduisent pas intégralement les raies considérées.

\section{REMERCIEMENT}

Nous remercions Valérie Van Grootel pour la disponibilité des données sur l'étoile HD149382 sur le Canadian Astronomy Data Centre, l'ISP pour le soutien financier et AFSIN pour le programme doctoral.

\section{RÉFÉRENCES}

[1] R. Aznar Cuadrado et C. S. Jeffery, « Physical parameters of sdB stars from spectral energy distributions », Astron. Astrophys., vol. 368, no 3, p. 994-1005, mars 2001, doi: 10.1051/00046361:20010068.

[2] A. U. Landolt, « UBVRI photometric standard stars around the celestial equator: updates and additions », Astron. J., vol. 137, no 5, p. 4186-4269, mai 2009, doi: 10.1088/0004-6256/137/5/4186.

[3] Gaia Collaboration et al., « Gaia Data Release 2: Summary of the contents and survey properties », Astron. Astrophys., vol. 616, p. A1, août 2018, doi: 10.1051/0004-6361/201833051.

[4] S. Geier, H. Edelmann, U. Heber, et L. MoralesRueda, « Discovery of a close substellar companion to the hot subdwarf star hd 149382 - the decisive influence of substellar objects on late stellar evolution », Astrophys. J., vol. 702, n 1, p. L96-L99, sept. 2009, doi: 10.1088/0004-637X/702/1/L96.

[5] U. Heber, « The atmosphere of subluminous B stars. II. Analysis of 10 helium poor subdwarfs and the birthrate of sdB stars. ", laap, vol. 155, p. 33-45, janv. 2086.

[6] G. Michaud, G. Alecian, et J. Richer, Atomic Diffusion in Stars, 1st ed. 2015. Cham: Springer International Publishing: Imprint: Springer, 2015.

[7] U. Heber, « Hot Subdwarf Stars », Annu. Rev. Astron. Astrophys., vol. 47, n 1, p. 211-251, sept. 2009, doi: 10.1146/annurev-astro-082708-101836.

[8] I. Kafando, F. LeBlanc, et C. Robert, « Detailed abundance analysis of five field blue horizontalbranch stars », Mon. Not. R. Astron. Soc., vol. 459, n ${ }^{\circ}$ 1, p. 871-879, juin 2016, doi: 10.1093/mnras/stw653.

[9] U. Heber, « Hot Subluminous Stars », Publ. Astron. Soc. Pac., vol. 128, n 966, p. 082001, août 2016, doi: 10.1088/1538-3873/128/966/082001.

[10] E. Niemczura, B. Smalley, et W. Pych, Éd., Determination of Atmospheric Parameters of B-, A-, F- and G-Type Stars: Lectures from the School of Spectroscopic Data Analyses, 1st ed. 2014. Cham: Springer International Publishing: Imprint: Springer, 2014.

[11] J.-F. Donati, « ESPaDOnS: An Echelle SpectroPolarimetric Device for the Observation of Stars at CFHT », in Solar Polarization, janv. 2003, vol. 307, p. 41.

[12] J.-F. Donati, C. Catala, J. D. Landstreet, et P. Petit, «ESPaDOnS: The New Generation Stellar SpectroPolarimeter. Performances and First Results », in Solar Polarization 4, déc. 2006, vol. 358, p. 362. 
[13] B. Baschek et J. Norris, « The OB-type subdwarf HD 149382 and the nature of the subdwarf B stars », Astrophys. J., vol. 209, p. 694, août 1975, doi: $10.1086 / 153739$.

[14] B. Baschek, P. Hoeflich, et M. Scholz, « The OB subdwarf Feige 66, a chemical-composition twin to HD 149382. ", laap, vol. 112, p. 76-82, août 1982.

[15] T. Lisker, U. Heber, R. Napiwotzki, N. Christlieb, D. Reimers, et D. Homeier, « Spectroscopic analysis of sdB stars from the ESO Supernova Ia Progenitor Survey », Astrophys. Space Sci., vol. 291, n 3, p. 351-358, 2004, doi: 10.1023/B.ASTR.0000044343.94946.51.

[16] U. Heber, K. Hunger, R. P. Kudritzki, et K. P. Simon, « NLTE analysis of SDO stars », Symp. Int. Astron. Union, vol. 105, p. 215-218, 1984, doi: 10.1017/S0074180900030916. 


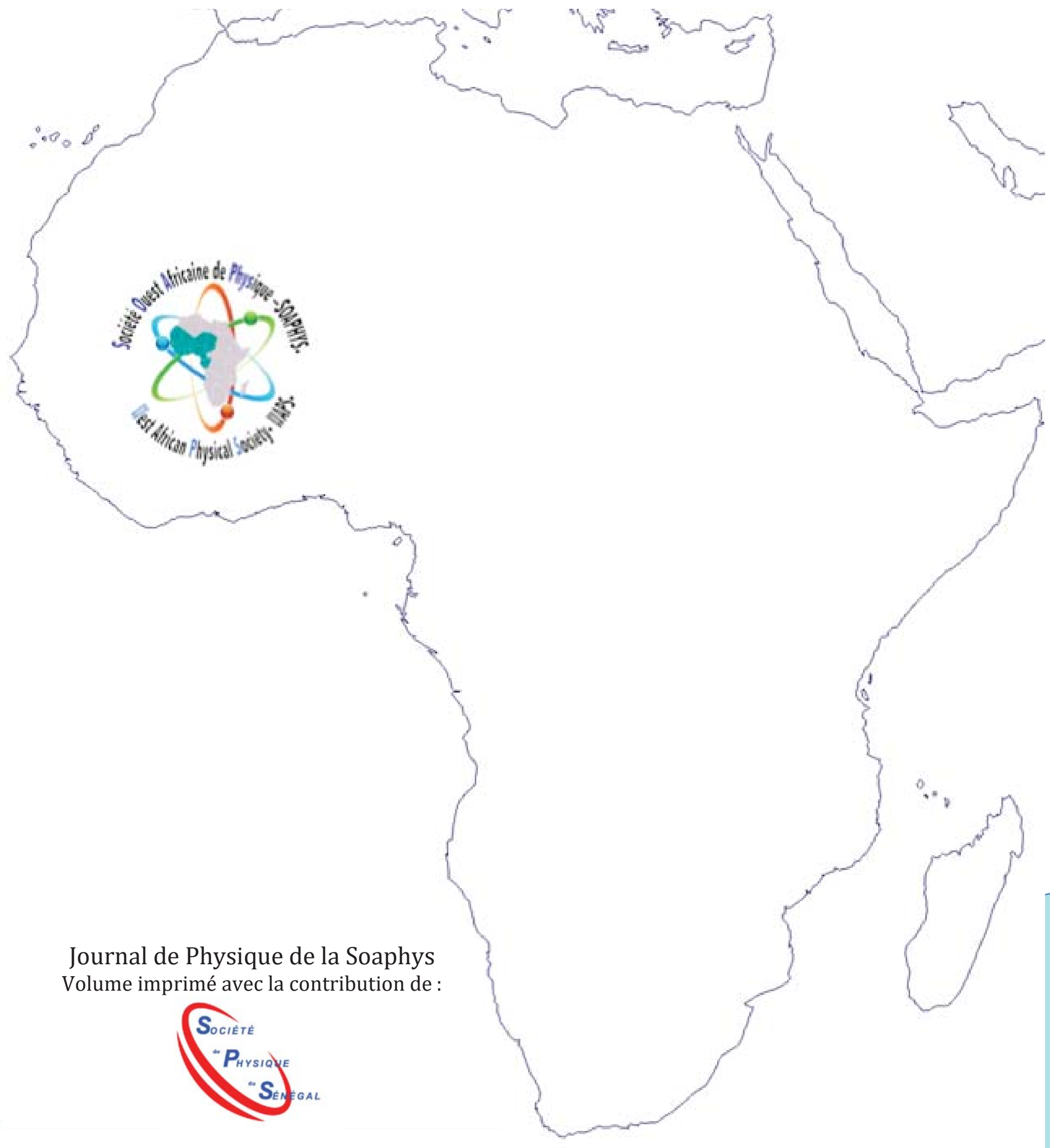

